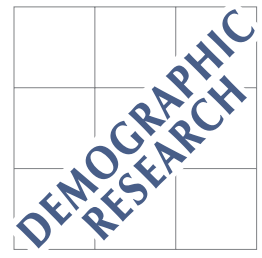

Demographic Research a free, expedited, online journal of peer-reviewed research and commentary in the population sciences published by the Max Planck Institute for Demographic Research Konrad-Zuse Str. 1, D-18057 Rostock · GERMANY www.demographic-research.org

DEMOGRAPHIC RESEARCH

VOLUME 21, ARTICLE 12, PAGES 341-366 PUBLISHED 15 SEPTEMBER 2009

http://www.demographic-research.org/Volumes/Vol21/12/

DOI: $\quad$ 10.4054/DemRes.2009.21.12

Research Article

Schelling's Segregation Model:

Parameters, scaling, and aggregation

Abhinav Singh

Dmitri Vainchtein

Howard Weiss

(c) 2009 Abhinav Singh et al.

This open-access work is published under the terms of the Creative Commons Attribution NonCommercial License 2.0 Germany, which permits use, reproduction \& distribution in any medium for non-commercial purposes, provided the original author(s) and source are given credit. See http://creativecommons.org/licenses/by-nc/2.0/de/ 


\section{Table of Contents}

$1 \quad$ Introduction $\quad 342$

$1.1 \quad$ Description of the Model 343

2 Schelling's segregation is a small city phenomenon 344

3 Simulations 346

$4 \quad$ Analysis 351

4.1 Measures of Aggregation 351

4.2 Global aggregation dependence on the neighbor comfort threshold $\mathbf{T} \quad 353$

4.2.1 $\mathbf{T}=\mathbf{3}$ : sparse clusters 353

4.2.2 $\mathbf{T}=4$ : compact clusters and mesoscale aggregation $\quad 355$

4.2.3 $\mathbf{T}=\mathbf{5}$ : final states with many unhappy agents 356

$\begin{array}{ll}4.3 & \text { Number of steps in the evolution } \\ \end{array}$

$5 \quad$ Final states with $\mathbf{N}=\mathbf{5 0}, \mathbf{N}=\mathbf{1 0 0}$ and $\mathbf{N}=\mathbf{2 0 0}$

$6 \quad$ Concluding remarks and acknowledgements 362

References $\quad 364$ 


\title{
Schelling's Segregation Model: Parameters, scaling, and aggregation
}

\author{
Abhinav Singh 1 \\ Dmitri Vainchtein ${ }^{2}$ \\ Howard Weiss ${ }^{3}$
}

\begin{abstract}
Thomas Schelling proposed a simple spatial model to illustrate how, even with relatively mild assumptions on each individual's nearest neighbor preferences, an integrated city would likely unravel to a segregated city, even if all individuals prefer integration. This agent based lattice model has become quite influential amongst social scientists, demographers, and economists. Aggregation relates to individuals coming together to form groups and Schelling equated global aggregation with segregation. Many authors assumed that the segregation which Schelling observed in simulations on very small cities persists for larger, realistic sized cities. We describe how different measures can be used to quantify the segregation and unlock its dependence on city size, disparate neighbor comfortability threshold, and population density. We develop highly efficient simulation algorithms and quantify aggregation in large cities based on thousands of trials. We identify distinct scales of global aggregation. In particular, we show that for the values of disparate neighbor comfortability threshold used by Schelling, the striking global aggregation Schelling observed is strictly a small city phenomenon. We also discover several scaling laws for the aggregation measures. Along the way we prove that in the Schelling model, in the process of evolution, the total perimeter of the interface between the different agents always decreases, which provides a useful analytical tool to study the evolution.
\end{abstract}

\footnotetext{
${ }^{1}$ School of Physics and Center for Nonlinear Science, Georgia Tech, USA, E-mail: abhinav.singh@ gatech.edu.

${ }^{2}$ Department of Mechanical Engineering, Temple University, USA; Space Research Institute, Moscow, Russia, E-mail:dmitri@temple.edu.

${ }^{3}$ School of Mathematics and Center for Nonlinear Science, Georgia Tech, USA, E-mail: weiss@math.gatech.edu.
} 


\section{Introduction}

In the 1970s, the eminent economic modeler Thomas Schelling proposed a simple spacetime population model to illustrate how, even with relatively minimal assumptions concerning every individual's nearest neighbor preferences, an integrated city would likely unravel to a segregated city, even if all individuals prefer integration (Schelling 1969; Schelling 1971a; Schelling 1971b; Schelling 2006). His agent-based lattice model has become quite influential amongst social scientists, demographers, and economists. Currently, there is a spirited discussion on the validity of Schelling-type models to describe actual segregation, with arguments both for (e.g., Young 1998; Fossett 2006), and against (e.g., Massey 1990; Laurie and Jaggi 2003), and a few authors have used and extended the Schelling model to address actual population data (Clark 1991; Bruch and Mare 2006; Benenson et al. 2006; Sander, Schreiber, and Doherty 2000; Clark and Fossett 2008). The few examples of quantitative analyses of such models are (Pollicott and Weiss 2001; Fossett 2006; Gerhold et al. 2008). Recently, Zhang (2004) proved analytically that, for certain wedge-like utility functions and with additional random noise, the equilibrium states possess a high degree of segregation.

Aggregation relates to individuals coming together to form groups or clusters, and Schelling equated global aggregation with segregation. Many authors assume that the striking global aggregation observed in simulations on very small ideal "cities" persists for large, realistic size cities. A recent paper (Vinkovic and Kirman 2006) exhibits final states for a small number of model simulations of a large city, and some final states that do not exhibit significant global aggregation. However, quantification of this important phenomenon is lacking in the literature, presumably due in part to the huge computational costs required to run simulations using existing algorithms. We develop highly efficient and fast algorithms that allow us to carry out many simulations for many sets of parameters and to compute meaningful statistics of the measures of aggregation.

The objective of this paper is to quantify the aggregation and unlock its dependence on city size, disparate neighbor comfort threshold, and population density. One of the measures is the total perimeter of a configuration: the total number of contacts between the agents of different kind, adjusted to the presence of empty spaces. We prove that as the system evolves, the total perimeter decreases at every step. This provides a useful analytical tool to study the evolution: it necessitates that the evolution of the Schelling model always converges to a limit configuration after a finite number of time steps. We identify distinct scales of global aggregation, and in particular, we show that for the values of disparate neighbor comfort threshold used by Schelling, the striking global aggregation Schelling observed is strictly a small city phenomenon. We also discover several remarkable scaling laws for the aggregation measures. 


\subsection{Description of the Model}

We expand Schelling's original model ${ }^{4}$ to a three parameter family of models. The phase space for these models is the $N \times N$ square lattice with periodic boundary conditions (opposite sides identified). We consider two distinct populations, that, in Schelling's words, refer to "membership in one of two homogeneous groups: men or women, blacks and whites, French-speaking and English speaking, officers and enlisted men, students and faculty, surfers and swimmers, the well dressed and the poorly dressed, and any other dichotomy that is exhaustive and recognizable" (Schelling 2006). We denote by $\mathrm{B}$ (black squares) and R (red squares, appear grey on black and white printing) these two populations. See Fig. 1. Together these agents fill up some of the $N^{2}$ sites, with $V$ remaining vacant sites (white squares). Each agent has eight nearest neighbors, corresponding to Moore, or Queen, neighborhood. Different neighborhoods were studied in different papers (see, e.g, Fossett 2006; Clark and Fossett 2008, where the size of the neighborhood was referred to as 'vision'). Fix a disparate neighbor comfort threshold $T \in\{0,1, \ldots, 8\}$, and declare that a B or R is happy if $T$ or more of its nearest eight neighbors are B's or R's, respectively. Else, it is unhappy.

Figure 1: $\quad$ A: A simulation of Schelling's original model with $N=8$;

B: Our simulation with $N=100$.
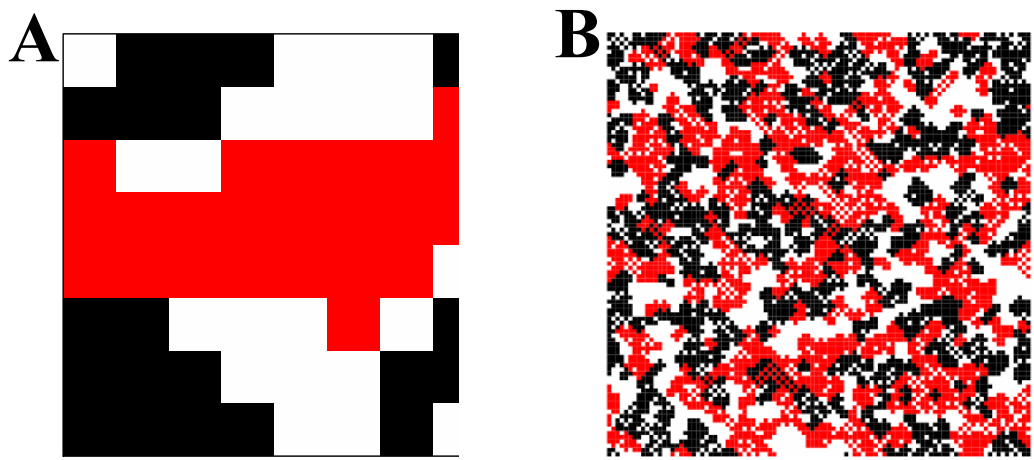

\footnotetext{
${ }^{4}$ Different authors frequently consider slightly different versions of Schelling's original model, i.e., different ways of moving boundary agents. All versions seem to exhibit the same qualitative behaviors, and thus we refer to the Schelling model.
} 
Demographically, the parameter $N$ controls the size of the city, $v=V / N^{2}$ controls the population density or the occupancy ratio (BusinessLocate 2009), and $T$ is an "agent comfort index" that quantifies an agent's tolerance to living amongst disparate nearest neighbors.

In choosing the algorithm of evolution we followed the protocol introduced in the original Schelling paper (Schelling 1969) and later used in Portugali, Benenson, and Omer (1994) and Benenson et al. (2006). We begin the evolution by choosing an initial configuration (described in Sect. 3) and randomly selecting an unhappy B and a vacant site surrounded by at least $T$ nearest $\mathrm{B}$ neighbors. Provided this is possible, interchange the unhappy B with the vacant site, so that this B becomes happy. We then randomly select an unhappy $\mathrm{R}$ and a vacant site having at least $T$ nearest neighbors of type R. Provided this is possible, we interchange the unhappy $\mathrm{R}$ with the vacant site, so that $\mathrm{R}$ becomes happy. We repeat this iterative procedure, alternating between selecting an unhappy $\mathrm{B}$ and an unhappy R, until a final state is reached, where no interchange is possible that increases happiness. For some final states, some (and in some cases, many) agents may be unhappy, but there are no allowable switches.

For the sake of completeness, we carried out simulations using other agent selection protocols, including random selection schemes. We observed no significant differences in the final states using the other selection schemes. This supports the claim in Young (1998) that the fine details of the evolution have negligible influence on the structure of the final states.

\section{Schelling's segregation is a small city phenomenon}

Schelling considered the cases city size $N=8$, neighbor comfort threshold $T=3$, and vacancy ratio $v=33 \%$. For $T=3$ or 4 , and $v=0$, a "checkerboard" configuration of B's and R's (imagine placing B's on the red squares and R's on the black squares of an actual checkerboard) is a final state, since all agents have four like nearest neighbors.

To generate his initial configurations, Schelling begins with a checkerboard configuration without periodic boundary conditions and randomly removes approximately one third of the B's and R's, keeping equal numbers of both agents (Schelling 2006). We refer to the result as a deleted checkerboard configuration. Removing these agents makes some of the remaining agents unhappy and drives the evolution. Several authors have observed that removing such a large percentage of agents is unnatural, but it is crucial to attain aggregation in Schelling's model. Removing fewer agents results in a final configuration close to the initial configuration. Finally, Schelling modifies the deleted checkerboard by randomly adding a total of 5 B's and 5 R's in vacant spaces. Such an initial configuration is assumed to be a proxy for a nearly integrated city. 
The final state of a typical run of Schelling's original model system is presented in Fig. 1A. Schelling performed many simulations by hand using an actual checkerboard, and observed that the final states presented a significant degree of global aggregation. $\mathrm{He}$ equated the global aggregation with segregation of a city.

In this paper, we investigate whether the global aggregation that Schelling observed for very small lattices persists for larger lattices. In Fig. 1B, we present a characteristic final state for our simulations with city size $N=100$. Comparing Figs. $1 \mathbf{A}$ and $1 \mathbf{B}$, one can see a striking qualitative difference between the two final states. While there is some local aggregation in the final state with $N=100$, there is no global aggregation. Visually inspecting this and other final states, one immediately sees that the global aggregation observed by Schelling is a small lattice phenomenon.

To quantify the difference in aggregation between a small city $(N=8)$ and a larger city $(N=100)$ we used a combination of two aggregation measures: the number of clusters in the final state of the model and the normalized average size of individual clusters (see Sect. 4.1 for a detailed description of these and several other measures of aggregation). We determine the normalized average size of a cluster by dividing the average size of a cluster by the total number of agents in the city. The latter determines the proportion of a city covered by an individual cluster and provides a way to compare aggregation between cities of different sizes. Figure 2 shows the mean values of these two aggregation measures in the final states of cities of two sizes $(N=8$ and 100). We compute the mean values of the two measures based on 100 trials for each choice of the vacancy ratio $v$ and neighbor comfort threshold $T=3$. We observe that the normalized average size of a cluster in the large city is smaller than one in a small city. This implies that an individual cluster in a large city covers a smaller proportion of the city as compared to a cluster in a smaller city. Most final states of the small city are segregated into two clusters for all choices of the vacancy ratio while the number of clusters in the large city increases from 22 for a city with $24 \%$ empty locations to 55 for a city with $33 \%$ empty locations. As we move from a small city to a large one, the relative size of a cluster in the final state decreases and the number of clusters increases. This shows that the large scale global aggregation observed by Schelling is strictly a small city phenomenon and does not occur for larger cities.

In Sections 3 through 5 we quantify the global aggregation using several new measures, and we analyze the structure of the final states for different values of neighbor comfort threshold $T$, vacancy ratio $v$, and city size $N$. 
Figure 2: $\quad$ Aggregation measures to distinguish between a small city $(N=8)$ and a large city $(N=100)$ for constant neighbor comfort threshold $T=3$ and different values of vacancy ratio $v$.

A: Normalized average size of an individual cluster.

B: Number of clusters in the final state of a city.
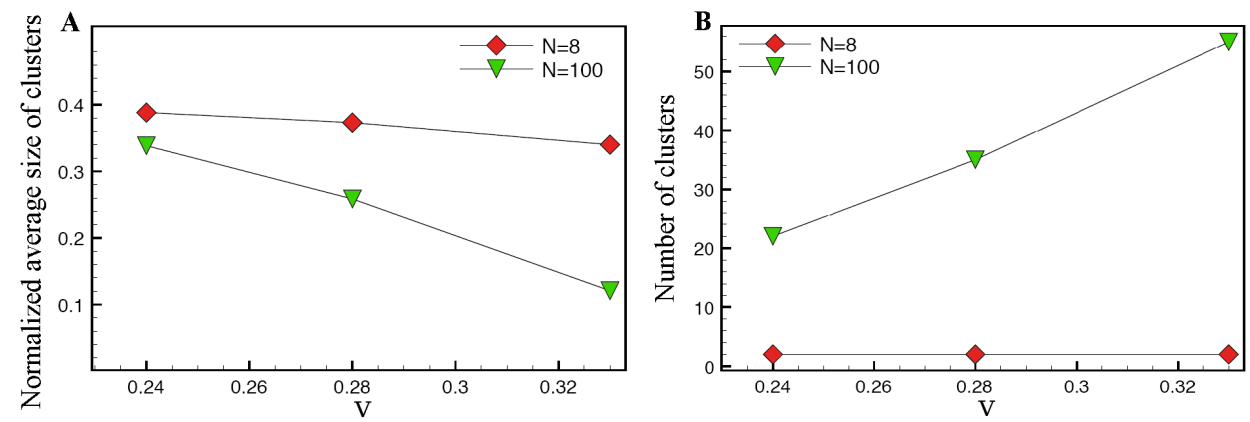

\section{Simulations}

We are the first authors to quantify aggregation in a large city based on a large number of simulations. We develop highly efficient algorithms to simulate the model and quantify the aggregation. We currently need approximately one minute to run a single simulation for a city of size $N=100$ and we ran more then nine thousand simulations for this manuscript. We achieve this boost in speed by coming up with innovative ways to determine the happiness of each agent and to find a suitable location for an unhappy agent. We exploit the ability of modern software packages such as NumPy (Oliphant 2006) to efficiently manipulate matrices; we can now compute properties of all agents in a city simultaneously thus escaping the slow process of dealing with each agent individually. The speed at which we find an unhappy agent in the city and a suitable location for it determines the speed of the simulation. Naively looking at each agent in the city to determine if it is unhappy and testing each empty location to determine if it is a suitable location for an unhappy agent, makes the simulation much slower. A much more efficient approach is to construct matrices highlighting the unhappy agents in the city and suitable locations for unhappy agents. The unhappiness of an agent and the suitability of a location are both based on the number of similar neighbors in the neighborhood of a given location. As an example of the efficiency gained by matrix methods, we outline the steps to determine 
the number of $\mathrm{R}$ agents surrounding each agent in the city simultaneously. In our 'city matrix', an $\mathrm{R}$ agent is represented by 1 , a $\mathrm{B}$ agent by -1 and an empty location by 0 . Therefore, the problem of determining the number of surrounding $\mathrm{R}$ agents is reduced to adding up the 1's in the neighborhood of each agent and ignoring the -1's. We ignore the -1 's by simply finding the absolute value of each element in the city matrix; this converts the -1's into 1's but leaves the 1's and 0's unchanged. We call this modified matrix the 'absolute value matrix'. When we add the city matrix and the absolute value matrix, all the -1 's are gone and the sum of all the elements gives the number of $\mathrm{R}$ agents in the 8-point neighborhood. Similar methods can be used to speed up the process of finding suitable locations for unhappy agents and computing aggregation measures.

We study the dynamics for large lattices and present our results mostly for city size $N=$ 100. Figures 2-7 are all based on $N=100$. In the last section, we discuss the cases $N=50$ and $N=200$, and show that $N$ greater than 100 does not lead to qualitatively or quantitatively different states and phenomena. We restrict our discussion to cities having an equal number of B's and R's. We will report the results on the dynamics with different proportions of B's and R's in a separate manuscript (Singh, Vainchtein, and Weiss 2009).

We consider neighbor comfort threshold $T=3,4,5$ and vacancy ratio $v$ between $2 \%$ and $33 \%$. The system does not evolve very much for other values of $T$ : for $T=1,2$ almost all of the agents are satisfied in most of the initial configurations, while for $T \geq 6$ there are almost no legal switches. Values of $v$ larger than $33 \%$ correspond to unrealistic environments. For each pair of parameters $T$ and $v$, we perform 100 simulations. This number of simulations was chosen to ensure a $95 \%$ confidence interval for parameter estimation. The Central Limit Theorem provides confidence intervals for the mean values of the aggregation measures.

We choose the initial configuration by starting with a checkerboard with periodic boundary conditions. Demographically, a checkerboard configuration is a maximally integrated configuration. We then randomly remove half the intended vacant locations $v / 2$ of both B's and R's (thus keeping equal numbers of both agents). We randomly permute agents in two $3 \times 3$ blocks. Alternatively, we could choose a completely random initial configuration. In general, except for small values of $v$, the final states are quantitatively similar to the ones obtained using the Schelling-like initial conditions.

In Figs. 3-5, we present characteristic final states for different values of $T$ and $v$. Visually, the aggregation in the final states with fixed $v$ are substantially different than in the final states with fixed $T$. 
Figure 3: $\quad$ Characteristic final states for neighbor comfort threshold $T=3$ for different vacancy ratios $v$ :
$\mathrm{A}: v=2 \%, \mathrm{~B}: v=6 \%, \mathrm{C}: v=10 \%, \mathrm{D}: v=15 \%$,
$\mathrm{E}: v=20 \%, \mathrm{~F}: v=24 \%, \mathrm{G}: v=28 \%, \mathrm{H}: v=33 \%$.
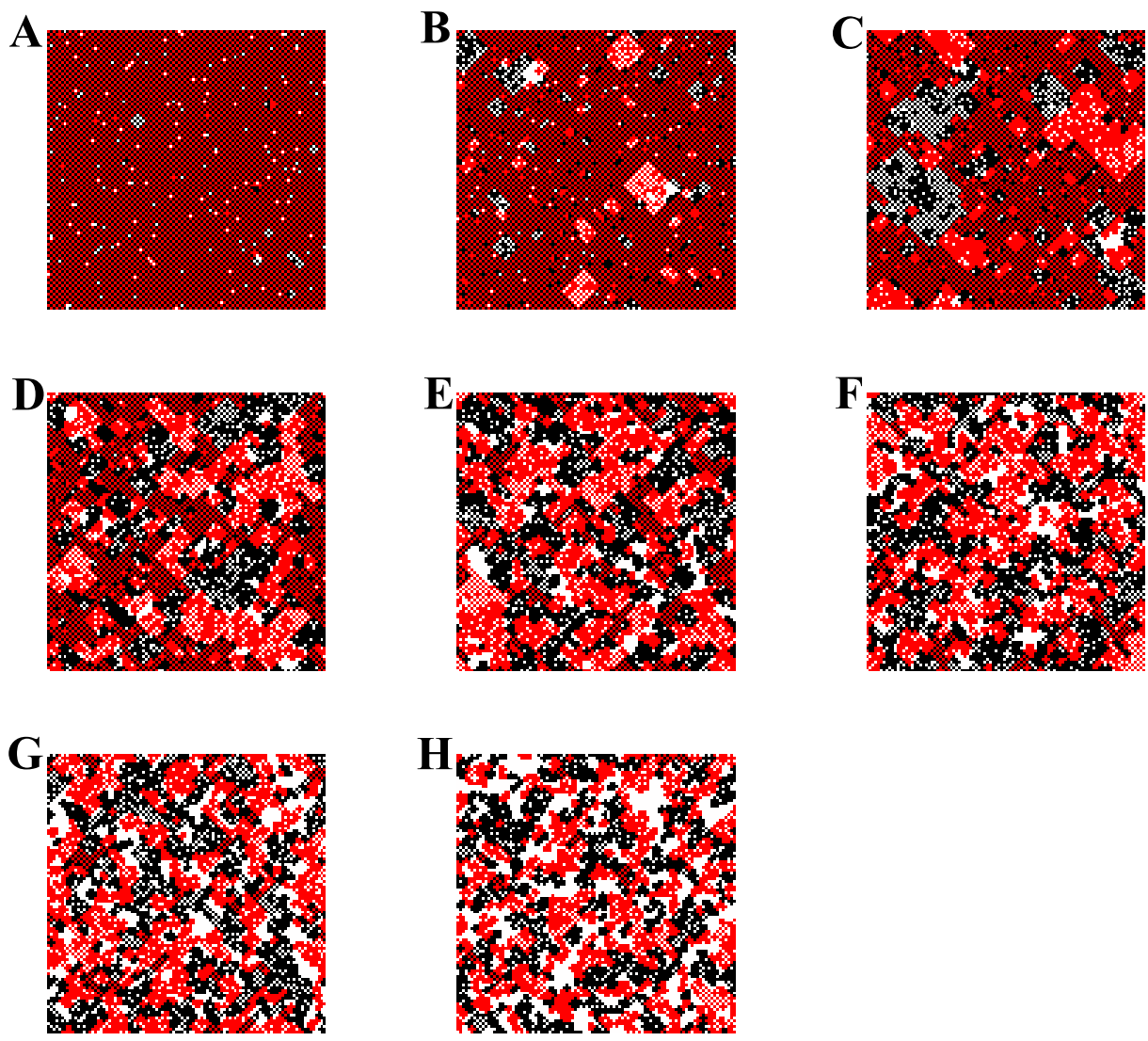
Figure 4: $\quad$ Characteristic final states for neighbor comfort threshold $T=4$ for different vacancy ratios $v$ :

$\mathrm{A}: v=2 \%, \mathrm{~B}: v=6 \%, \mathrm{C}: v=10 \%, \mathrm{D}: v=15 \%$,

$\mathrm{E}: v=20 \%, \mathrm{~F}: v=24 \%, \mathrm{G}: v=28 \%, \mathrm{H}: v=33 \%$.
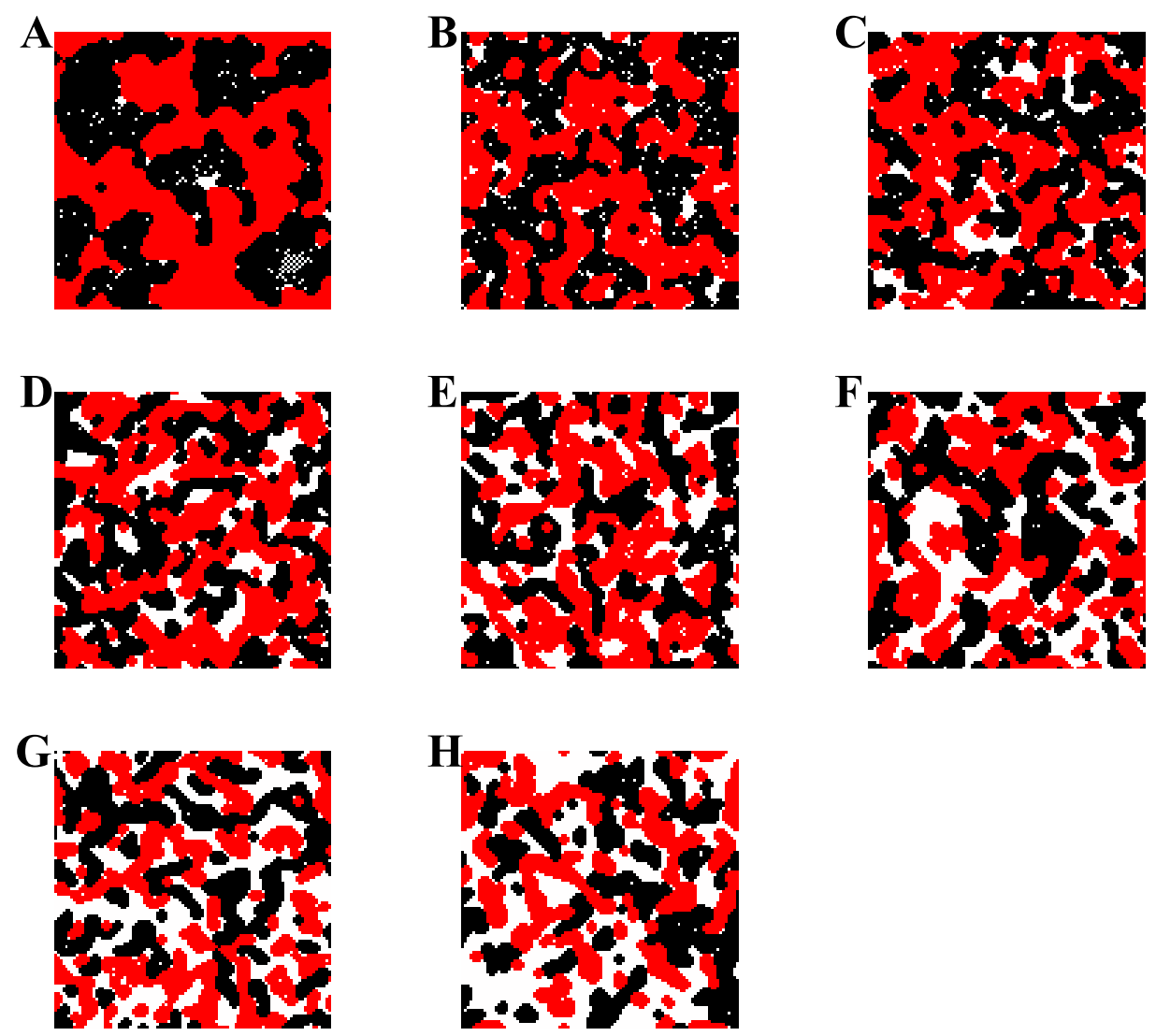
Figure 5: $\quad$ Characteristic final states for neighbor comfort threshold $T=5$ for different vacancy ratios $v$ :
$\mathrm{A}: v=2 \%, \mathrm{~B}: v=6 \%, \mathrm{C}: v=10 \%, \mathrm{D}: v=15 \%$,
$\mathrm{E}: v=20 \%, \mathrm{~F}: v=24 \%, \mathrm{G}: v=28 \%, \mathrm{H}: v=33 \%$.
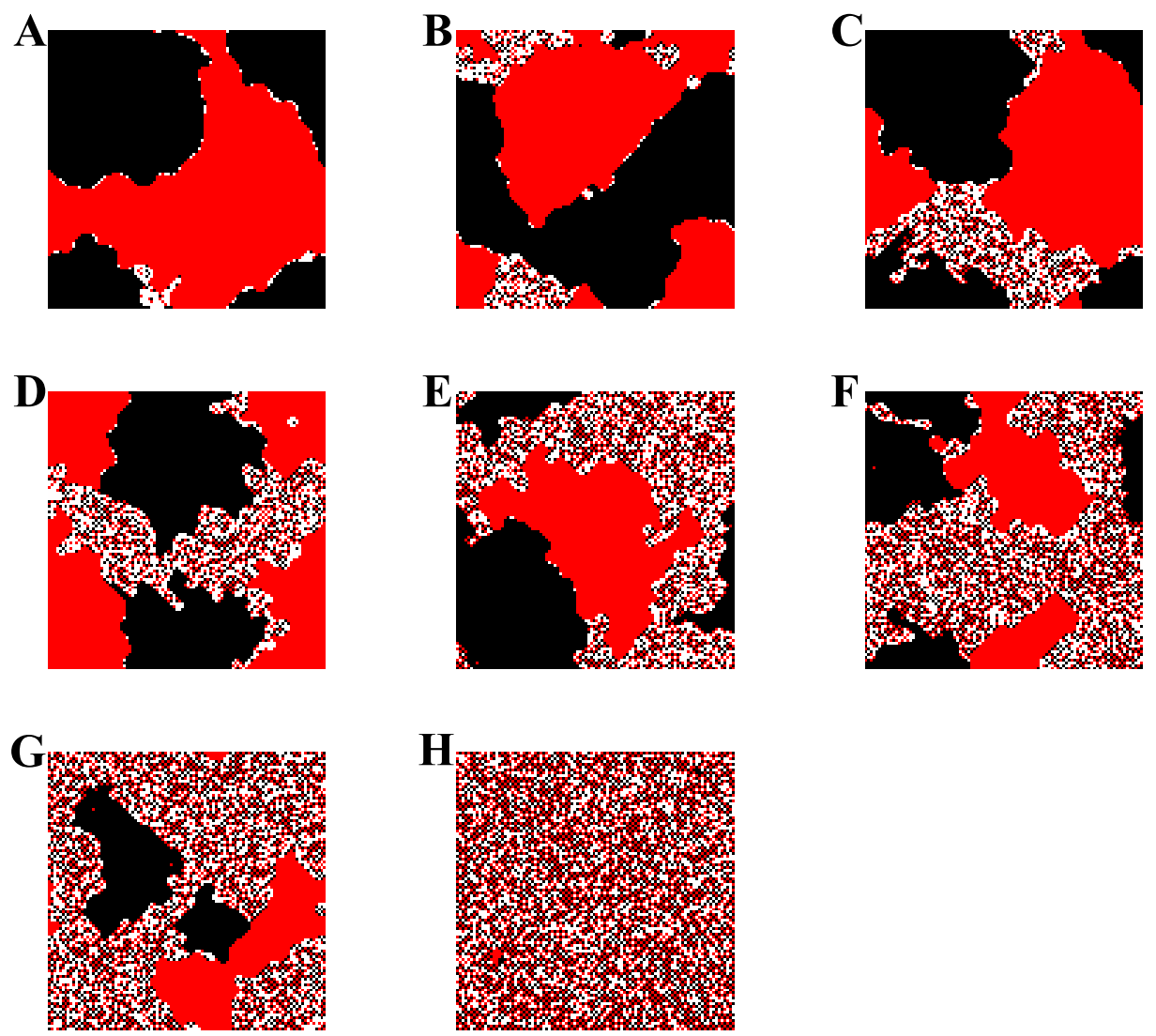


\section{Analysis}

\subsection{Measures of Aggregation}

In Figs. 3-5, aggregation appears to be a multifaceted phenomenon. One can observe that the states are visually quite different. However, to draw any quantitative conclusions and to arrive to any meaningful demographic observations, we need several measures (or indices) to describe the states. We begin the current section by defining several measures of aggregation that enable us to quantify this observation. In his papers, Schelling used two measures of aggregation:

1. The ratio of unlike to like neighbors that is called the $[u / l]$-measure. For a site on the lattice with coordinates $(i, j)$ we define:

$$
[u / l]_{i, j}=\frac{q_{i, j}+w_{i, j}}{s_{i, j}}
$$

where $s_{i, j}, q_{i, j}$, and $w_{i, j}$ are the number of like, unlike, and vacant neighbors of the agent located at $(i, j)$, respectively. We define the sparsity $\langle[u / l]\rangle$ of a cluster by averaging the $[u / l]$-measure over the given cluster.

2. The number of agents that have neighbors only of the same kind (note that this definition excludes the vacant spaces as well). The abundance of such agents indicate the presence of large, "solid" clusters. This quantity is the most useful in quantifying between the states with $T=3$ and $T=4$. We call the latter quantity seclusiveness and denote by $N_{0}$.

Since the publication of Schelling's papers, sociologists have devised new measures to quantify different aspects of segregation, including: evenness, exposure, clustering, concentration and centrality (Duncan and Duncan 1955; Massey and Denton 1988; Massey, White, and Phua 1996). Exposure relates to the degree of contact between agents of different kinds and clustering relates to the degree of contiguity among agents of one kind. In this paper we concentrate on the exposure and clustering aspects of segregation. Along with Schelling's two measures of exposure, we introduce an additional measure of exposure, as well as two measures of clustering.

3. The adjusted perimeter per agent $p$ of the interface between the different agents suitably adjusted for the vacant spaces. The perimeter $P$ is defined as twice the total number of R-B connections plus the total number of connections between $\mathrm{R}$ and $\mathrm{B}$ agents with vacant spaces. Demographically, the adjusted perimeter, $p=P / N^{2}$, is the average number of contacts an agent has with the opposite kind or with vacant sites. In the segregation literature, the perimeter is related to the exposure index (see, e.g., Massey and Denton 1988). 
A key observation is that $p$ is a Lyapunov function, i.e., a function defined on every configuration which is strictly decreasing along the evolution of the system until it reaches a final state. Thus the system evolves to minimize the adjusted interface between the $\mathrm{R}$ and $\mathrm{B}$ agents. The final states are precisely the local minimizers of the Lyapunov function, subject to the threshold constraint. This Lyapunov function is also the Hamiltonian for a related spin lattice system related to the Ising model (Simon 1993). Such a notion of $p$ was motivated by analogies of these models with the physics of foams. Note that for wedge-like utility functions, such as the ones considered in (Zhang 2004), $p$ is not a Lyapunov function, even in the absence of noise.

Let us show that in the process of evolution every legal switch makes $P$ smaller. Suppose we switch an $\mathrm{R}$ and a $\mathrm{V}$. Before the switch, suppose $\mathrm{R}$ had $B_{1}, R_{1}$, and $V_{1}$, of $\mathrm{B}, \mathrm{R}$, and $\mathrm{V}$ neighbors, respectively. Similarly, the numbers for the $\mathrm{V}$ agent are $D_{2}, R_{2}$, and $V_{2}$. Then the value of $P$ before and after the switch are:

$$
P_{\text {initial }}=2 B_{1}+V_{1}+B_{2}+R_{2} ; \quad P_{\text {final }}=2 B_{2}+V_{2}+B_{1}+R_{1} .
$$

Thus,

$$
P_{\text {final }}-P_{\text {initial }}=B_{2}+V_{2}+R_{1}-\left(B_{1}+V_{1}+R_{2}\right) .
$$

Taking into account that $B_{1}+V_{1}+R_{1}=B_{2}+V_{2}+R_{2}=8$, we arrive at

$$
P_{\text {final }}-P_{\text {initial }}=2\left(R_{1}-R_{2}\right)<0 .
$$

Similarly, if the switch between $\mathrm{R}$ and $\mathrm{B}$, we have

$$
P_{\text {final }}-P_{\text {initial }}=2\left(R_{2}-R_{1}\right)+2\left(B_{1}-B_{2}\right)<0 .
$$

The main consequence of the presence of a Lyapunov function is that it guarantees the convergence of the Schelling model to a final steady state. Moreover, since $P$ decreases by at least 2 on every switch and $P$ cannot be negative, there can only be finitely many moves before the algorithm converges to an equilibrium state.

4. The scale, or maximum diameter of the connected components (which we henceforth call clusters) $L$. The measure $L$ is defined as the side length of the smallest square needed to cover every cluster. For configurations consisting of mostly compact clusters, the maximum diameter, $L=\max \left(L_{i}\right)$, defines the scale of aggregation. Depending on the value of $L$, we can say that, in terms of the analogies suggested in (Clark and Fossett 2008), the aggregation happens on the neighborhood (small $L$ ), school district (medium $L$ ), or global, city-wide (large $L$ ) scale. Checkerboard configurations and configurations consisting of compact clusters are two extremes; for the former $L=N$. The diameter of a cluster can be easily computed as the larger between the number of rows that contain an agent belonging to the cluster and the number of columns that contain an agent belonging to the cluster. 
5. The total number of clusters in a configuration $N_{C}$. This intuitively appealing measure of aggregation is useful to describe final states having mostly large compact clusters. For such systems, $N_{C}$ and $L$ are the quantities that attract the viewer's attention first. To see its limitation, observe that "the maximally integrated" checkerboard configuration with $v=0$ has just $1+1=2$ clusters. The reason for that is that if two squares are considered to belong to the same cluster if they touch by a side or a vertex, clusters may be intermingled. The quantity $N_{C}$ is the most useful for configurations consisting of compact clusters of a similar size. To study configurations such as the final states for $T=5$, a more useful quantity is the number of clusters that have greater then, say, $M_{\max } / 10$ agents, where $M_{\max }$ is the number of agents in the largest cluster of a given kind.

In Fig. 6, we plot average values of aggregation measures (2)-(5) introduced above for the final states with $T=3,4,5$ and several values of $v$. The linear relationships of these disparate aggregation measures on population density seem remarkable. Often there is some deeper meaning behind such linear scaling, such as critical exponents in phase transitions, that may lead to construction of theoretical explanation of the phenomena.

\subsection{Global aggregation dependence on the neighbor comfort threshold $T$}

From Figs. 3-5, one observes that:

1. the final states with neighbor comfort threshold $T=3$ are very sparse, with a great deal of interweaving between both kinds of agents and vacant spots;

2. the final states with $T=4$ consist of compact clusters (that look like solid objects); and, finally,

3. the final states with $T=5$ consist of one (for each type) huge cluster together with a small number of remaining agents scattered around.

Varying the density $v$ does not radically alter the qualitative structure of the final states. We now quantify the aggregation for each value of $T$, as $v$ varies between $2 \%$ and $33 \%$.

\subsection{1 $T=3$ : sparse clusters}

In Fig. 3 we present typical final states for neighbor comfort threshold $T=3$ and different values of vacancy ratio $v$. Unlike the final states for Schelling's original model with city size $N=8$ (see Fig. 1A) that consists of just one or two separate domains of R and B agents, the final states for $N=100$ and $v=33 \%$ (Fig. 3H) contain many clusters. The striking qualitative difference is also quantified by the relatively large values of the normalized perimeter, $p$ (see Fig. 6D). 
Figure 6: $\quad$ Statistics of four key measures of aggregation of final states for neighbor comfort threshold $T=3$ (red triangles), $T=4$ (green squares), and $T=5$ (blue circles) for different vacancy ratio $v$ :
A The scale of aggregation $L$;
B The number of clusters $N_{C}$;
C The number of agents with eight like nearest neighbors $N_{0}$;
D Normalized perimeter $p$.
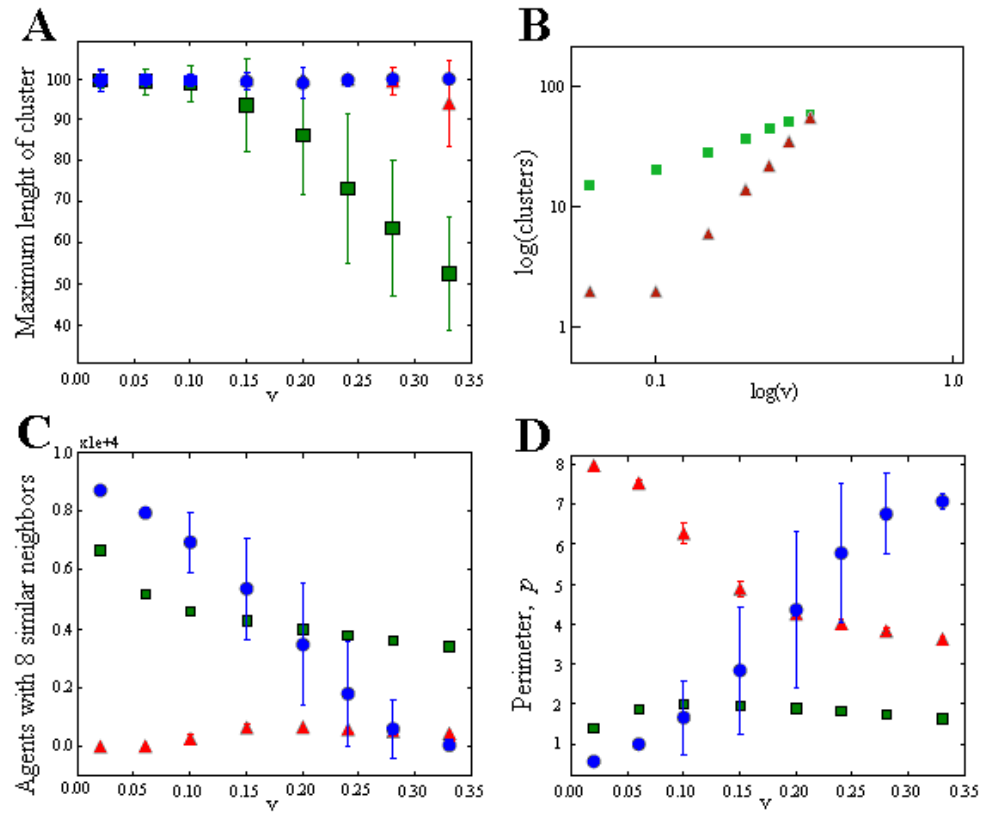

The sparsity of the final states with $T=3$ is due, in part, to large blocks of the initial checkerboard configuration that remain unchanged during the evolution. We call this phenomenon the super-stability of the checkerboard. Every agent is not just happy, but has one like neighbor to "spare". Thus, it takes a large perturbation to make a given agent move and, therefore, only agents close to the initially perturbed sites move. Consequently, Schelling required a large density of vacant spaces $v(33 \%)$ to overcome checkerboard super-stability. Following the panels of Fig. 3, one can see that as $v$ decreases, larger and larger parts of the initial configuration remain unchanged during the evolution.

We observe that for neighbor comfort threshold $T=3$, larger values of vacancy ratio $v$ 
result in larger clusters, and thus lead to greater global aggregation. The number of clusters in the final states, $N_{C}$, decreases as $v$ decreases (Fig. 6B) and the dependence is almost cubic. The value for the slope in Fig. $6 \mathbf{B}$ corresponding to $T=3$ is 2.86 and the value 3 is well within the error bars. We can group the cubic dependence of the number of clusters and the previously noted linear dependence of aggregation measures on population density under the category of scaling laws. We use the term "scaling law" in the same sense as it is used in physics and biology: as a particularly simple relation (e.g., linear, cubic, etc.) between two important variables. Often there is deep science behind scaling laws (such as critical exponents in phase transitions and species-area relationships in ecology) and attempts to explain them often lead to a theoretical explanation of the phenomena.

The seclusiveness measure, $N_{0}$, is a monotonically decreasing function of $v$ : as $v$ decreases, the final state approaches the checkerboard and, naturally, almost all the agents have some contacts with other agents. Similarly, the smaller the value of $v$, the larger the normalized perimeter, $p$. The values of $p$ around 4 for large values of $v$ indicate that most of the agents have in their 8-point neighborhoods around $2-3$ vacancies and 1 or 2 agents of a different kind. This conclusion is supported by the visual inspection of Fig. 3F-H. Therefore, the low comfort threshold results for an individual agent in the presence of the vacancies in the neighborhood, rather than the agents of a different kind.

\subsection{2 $T=4$ : compact clusters and mesoscale aggregation}

In Fig. 4 we present typical final states for neighbor comfort threshold $T=4$ and different values of vacancy ratio $v$. Increasing $T$ from 3 to 4 eliminates the checkerboard superstability phenomenon and results in strikingly different structures of aggregation in final states. Namely, every final state consists of relatively small number of compact clusters, that clearly depends on $v$.

For relatively large values of $v$, such final states exhibit mesoscale aggregation and, for small values of $v$, macroscale aggregation. There seems to be no canonical way to separate the two types of aggregation. Our criterion is to define the transition when the size of the largest cluster, $L$, becomes equal to $N$.

We find two measures that clearly differentiate the global clustering of the final states for $T=3$ and $T=4$. First, the final states have very different perimeters (see Fig. 6D). Second, for $T=3$, the clusters are very sparse, while for $T=4$, the clusters are compact. A natural way to quantify this is to use $N_{0}$, whose statistics we present in Fig. $6 \mathrm{C}$. The measure $N_{0}$ is a monotonically increasing function of $v$. Note, that even for a relatively mild neighbor comfort threshold $T=4$, almost $40 \%$ or more of the population have only the like neighbors (for $T=3$ this number is of order of $10 \%$ )! It means that many agents 
report living in a "dense" conditions (no vacancies in the neighborhood), while the vacant spots create patches of their own.

One can see in Fig. 6D that the characteristic values of $p$ are at most 2 for all the values of $v$. It indicates that most of the agents have in their 8-point neighborhoods just $1-2$ vacancies and no agents of a different kind. This conclusion is supported by the visual inspection of Fig. 4F-H. Thus, for the low comfort threshold $T=4$ there are almost no contacts between the agents of a different kind.

In addition to the seclusiveness, $N_{0}$, we quantify the differences in the final states for neighbor comfort threshold $T=4$ with vacancy ratio $v$ ranging from $v=33 \%$ down to $v=2 \%$, with three measures: the number of clusters, $N_{C}$, the scale of aggregation, $L$, (Fig. 6). By providing opportunities for increasingly "easier satisfaction," one might believe that decreasing $v$ increases the values of number of clusters $N_{C}$. In other words, when there are a lot of vacancies, agents have many choices and it leads to appearance of many small "islands". Our study confirms this, and the dependence is remarkably linear. The value for the slope in Fig. $6 \mathbf{B}$ corresponding to $T=4$ is 0.89 and the value 1 is well within the error bars. Specifically, for typical final states with $T=4, v=33 \%$ (Fig. $4 \mathbf{H}$ ) $N_{C}$ is relatively high; for $T=4, v=15 \%, N_{C}$ is smaller (Fig. 4D) and the clusters on average are bigger; finally, states with $T=4, v=2 \%$ contain only a few compact clusters of either type that stretch across the whole lattice $(L=100)$. In general, as $v$ decreases, $L$ increases almost linearly (see Fig. 6A).

Thus for $T=3$ and $T=4$, the increase in $v$ leads to the opposite effects: they increase and decrease the level of global aggregation, respectively.

\subsection{3 $T=5$ : final states with many unhappy agents}

For small vacancy ratio $v$, the dynamics with neighbor comfort threshold $T=5$ always results in a final state achieved after just a few switches, and consists of mostly unhappy agents with no vacant space to where they could move. However, a slight modification of the selection algorithm to allow direct R-B switches (similar to the selection algorithms in (Pollicott and Weiss 2001; Weisbuch et al. 2002; Zhang 2004)), results in significant global aggregation and drastically reduces the number of unhappy agents, although not eliminating them entirely. The presence of unhappy agents in the final states is a new phenomenon, which we do not observe in simulations for $T=3$ (while such configurations theoretically exist, they are extremely unlikely) and is much less pronounced for $T=4$.

In Fig. 5 we present typical final states for neighbor comfort threshold $T=5$ and different values of vacancy ratio $v$ with modified selection algorithm. A typical $T=5$ final state consists of one big cluster for each kind of agent and the rest of the agents are unhappy and scattered around. As the value of $v$ decreases, the number of unhappy agents in final states $v$ decreases (Fig. 7A), and the size of a single (for each type) major cluster 
increases (see Fig. 7B). Another clear indication of the growth of the main cluster is the increase of the number of agents with 8 similar neighbors, $N_{0}$, illustrated in Fig. $6 \mathbf{C}$.

The globally aggregated final states (small values of $v$ ) with $T=5$ (with modified selection) and $T=4$ (with Schelling selection) appear similar in terms of the number of large clusters and the scale of aggregation, $L$ (Figs. 5A and 4A). However, there is a large difference in their adjusted perimeter $p$ : it is much smaller for neighbor comfort threshold $T=5$ (see Fig. 6D). There are two reasons for smaller $p$. First, the clusters are more "circular", thus reducing the perimeter-to-area ratio. Second, there are almost no vacant spots inside the clusters for $T=5$ : almost all the vacant spots are located at the boundary between the $R$ and $B$ clusters.

Figure 7: $\quad$ Statistics of the final states with neighbor comfort threshold $T=5$ :

A: The average number of unhappy agents in final states; B: The average number of the agents in the two big clusters.
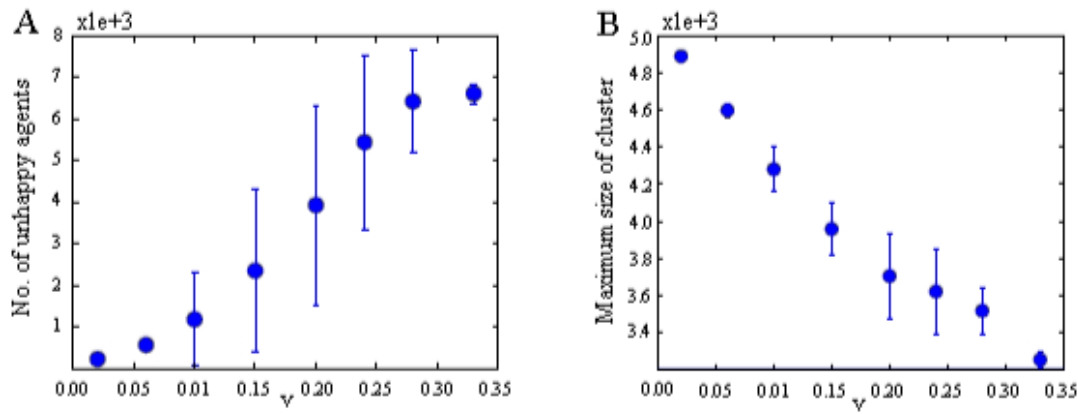

The average number of unhappy agents in final states for different values of $T$ and $v$ is presented in Fig. 7A. It is remarkable that the average number of unhappy agents is almost a linear function of vacancy ratio $v$, between $v=10 \%$ (where they constitute approximately $10 \%$ ) and $v=30 \%$ (where they constitute approximately $33 \%$; in other words, almost every agent). While the existence of unhappy agents in the final state does not significantly increase the perimeter $p$ of the final states, it greatly inflates the total number of clusters $N_{C}$.

\subsection{Number of steps in the evolution}

To illustrate the dynamics of the evolution, we computed the average number of switches required for the final state to be achieved for characteristic values of neighbor comfort 
threshold $T$ and vacancy ratio $v$. For $T=3, v=33 \% ; T=4, v=33 \% ; T=4$, $v=2 \%$; and $T=5, v=2 \%$ the average number of steps are $3596,5192,5573,4422$, respectively. The switches for $T=5, v=2 \%$ that included both $\mathrm{R}$ and $\mathrm{B}$ agents are counted twice. The most striking feature is that it takes significantly fewer switches to achieve the final state for $T=5$ than for $T=4$. In Fig. 8 we present the distribution of the number of jumps for different agents.

\section{Final states with $\mathrm{N}=50, \mathrm{~N}=100$ and $\mathrm{N}=200$}

To illustrate the dependence of the final states of city size $N$, we performed 100 simulations for $N=50, N=100$ and $N=200$. In Figs. 9-11 we present characteristic final states for $N=50, N=100$, and $N=200$, respectively. One can see that the figures for $N=50$ and $N=200$ are qualitatively very similar to Fig. 10 .

In Fig. 12 we present characteristic plots of two of the aggregation measures (namely the perimeter and the number of agents with only like neighbors) for city size $N=50$, $N=100$, and $N=200$. One can see that all three columns are very similar to each other.

Figure 8: $\quad$ Statistics of the number of agent jumps.
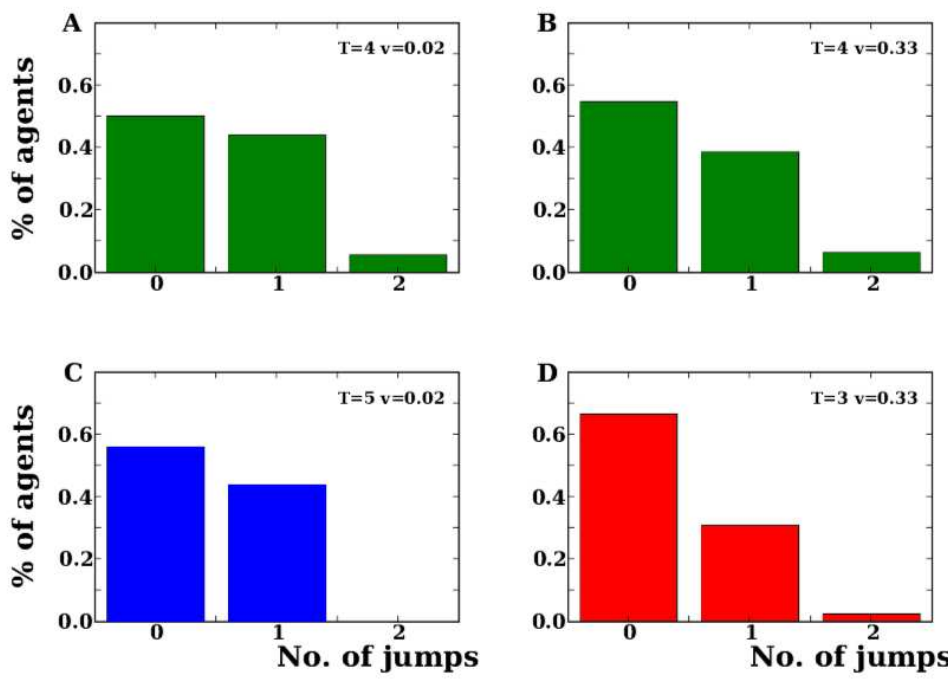
Figure 9: Characteristic final states for $N=\mathbf{5 0}$ and different values of neighbor comfort threshold $T$ and vacancy ratio $v$ :
$\mathrm{A}: T=3, v=2 \%, \mathrm{~B}: T=3, v=15 \%, \mathrm{C}: T=3, v=33 \%$,
$\mathrm{D}: T=4, v=2 \%, \mathrm{E}: T=4, v=15 \%, \mathrm{~F}: T=4, v=33 \%$,
G: $T=5, v=2 \%$, H: $T=5, v=15 \%$, I: $T=5, v=33 \%$.

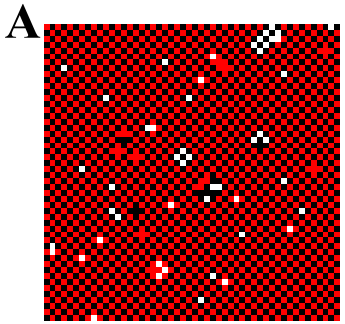

D
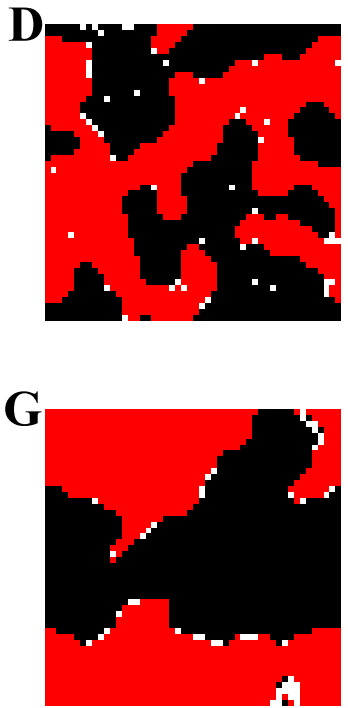

B

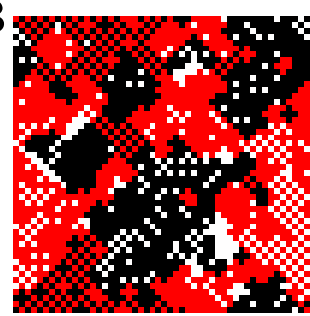

E

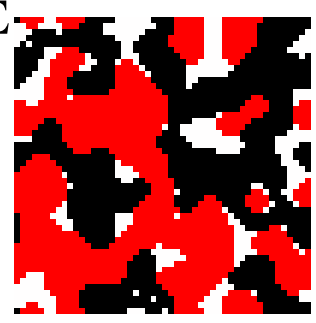

$\mathbf{H}$

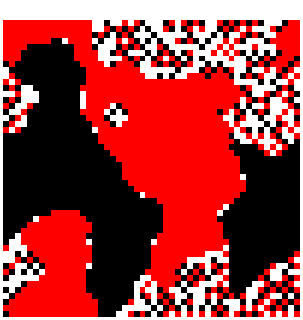

C

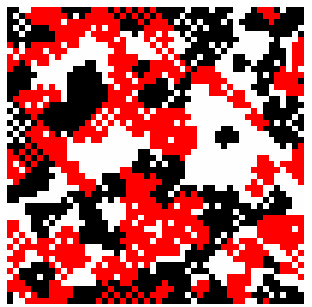

F
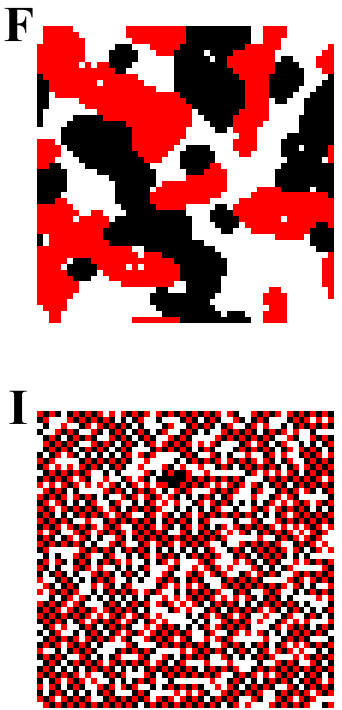
Figure 10: Characteristic final states for $N=100$ and different values of neighbor comfort threshold $T$ and vacancy ratio $v$ :
$\mathrm{A}: T=3, v=2 \%, \mathrm{~B}: T=3, v=15 \%, \mathrm{C}: T=3, v=33 \%$,
$\mathrm{D}: T=4, v=2 \%, \mathrm{E}: T=4, v=15 \%, \mathrm{~F}: T=4, v=33 \%$,
G: $T=5, v=2 \%$, H: $T=5, v=15 \%$, I: $T=5, v=33 \%$.
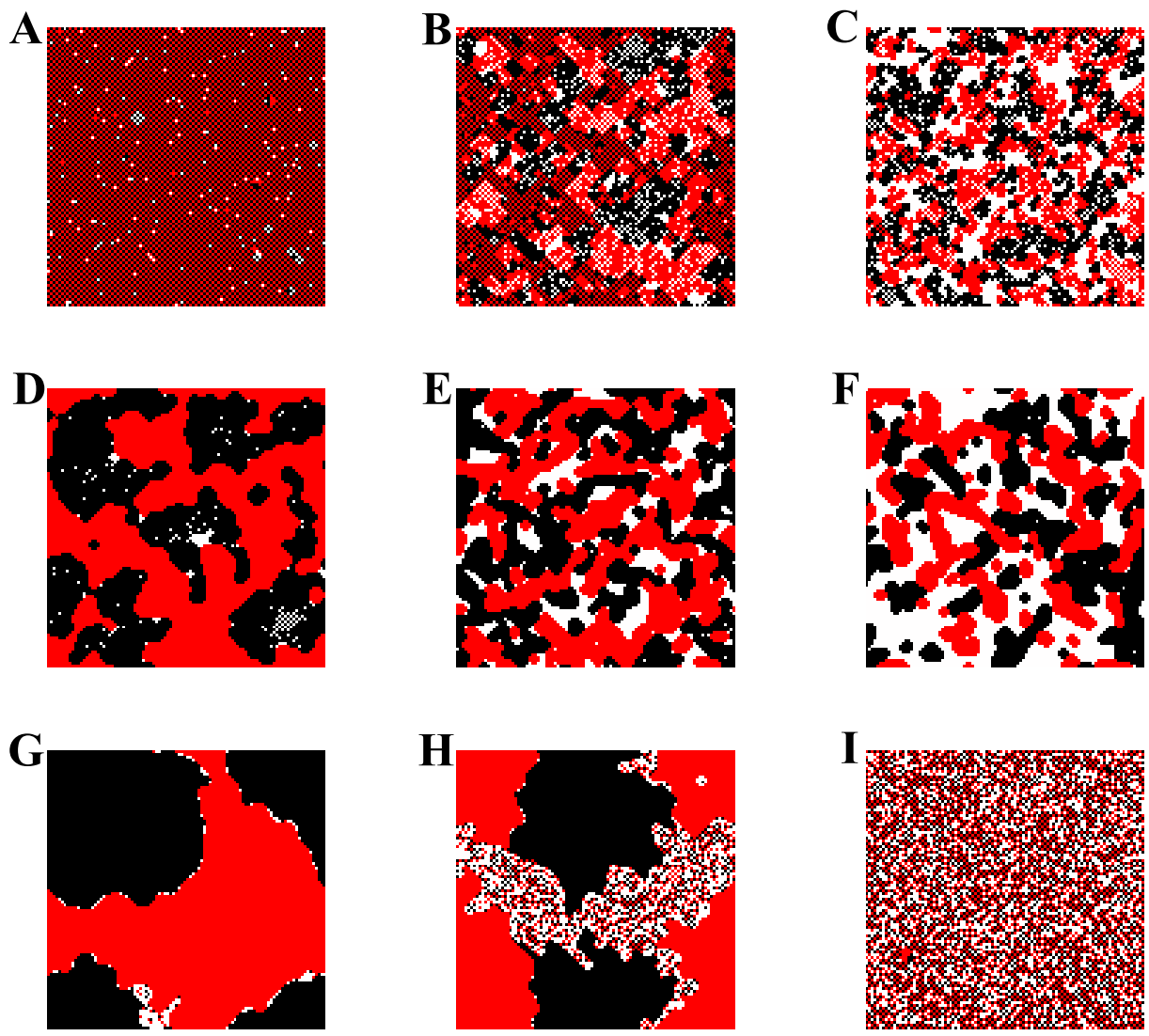
Figure 11: Characteristic final states for $N=200$ and different values of neighbor comfort threshold $T$ and vacancy ratio $v$ :

$$
\begin{aligned}
& \text { A: } T=3, v=2 \%, \mathrm{~B}: T=3, v=15 \%, \mathrm{C}: T=3, v=33 \% \\
& \mathrm{D}: T=4, v=2 \%, \mathrm{E}: T=4, v=15 \%, \mathrm{~F}: T=4, v=33 \%, \\
& \text { G: } T=5, v=2 \%, \mathrm{H}: T=5, v=15 \%, \mathrm{I}: T=5, v=33 \%
\end{aligned}
$$
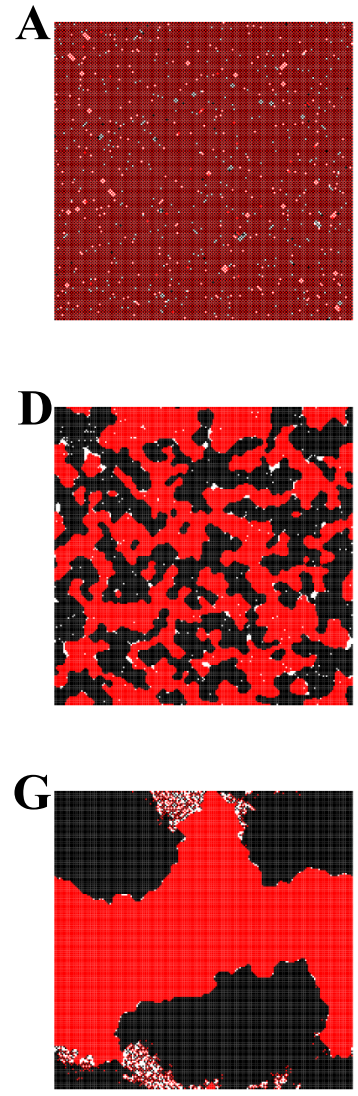

B

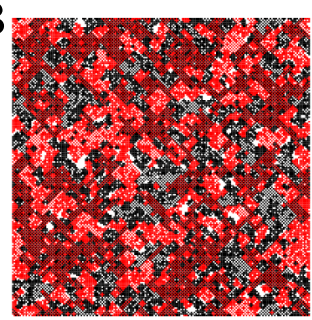

$\mathbf{E}$

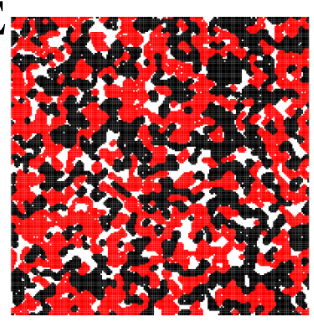

$\mathbf{H}_{\mathbf{r}}$

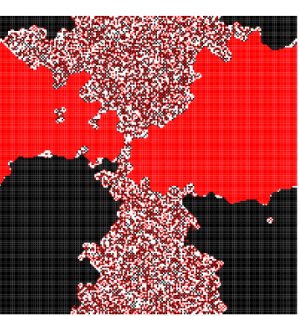

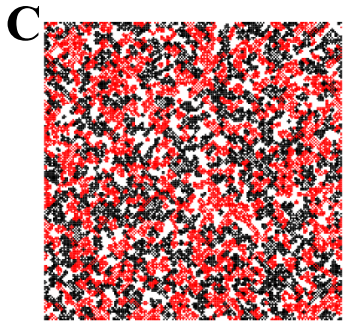

$\mathbf{F}$

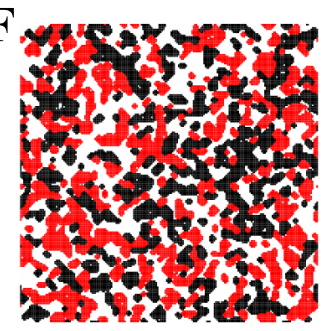

I

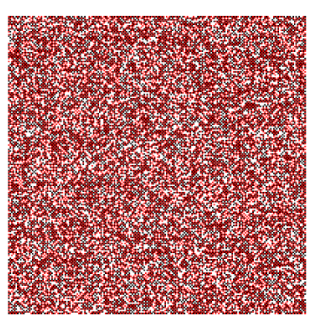


Figure 12: Characteristic values of the perimeter (top row) and the number of agents with only like neighbors (bottom row) for $N=\mathbf{5 0}$ (left column), $N=100$ (middle column), and $N=200$ (right column) different values of neighbor comfort threshold $T$ and vacancy ratio $v$; in every frame, $T=3$ (red triangles), $T=4$ (green squares), and $T=5$ (blue circles).
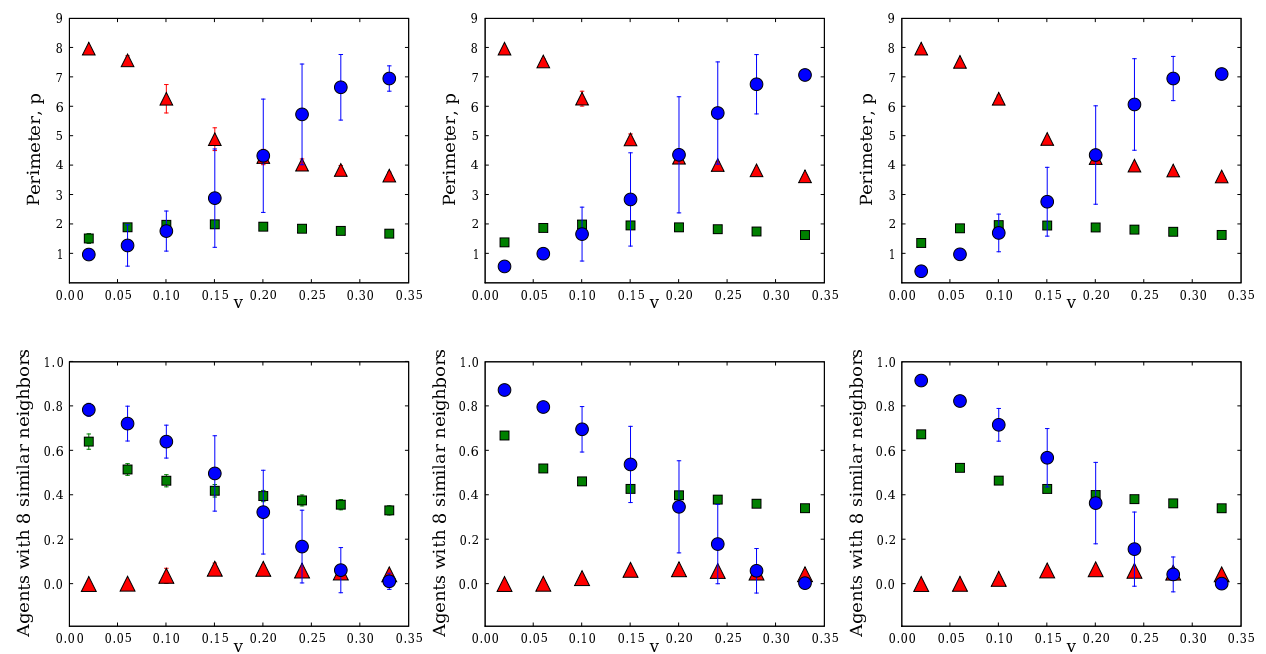

\section{Concluding remarks and acknowledgements}

In this paper, we quantify the dependence of city aggregation in the Schelling model on city size, disparate neighbor comfort threshold, and population density. We make two methodological innovations: we devise new measures to quantify the aggregation in the Schelling model and we develop new fast algorithms to simulate a large city in the model. We ran thousands of simulations for a large city, something which has never been done before, and compiled accurate statistics of aggregation in a large city based on these simulations. We find that the striking global aggregation Schelling observed for disparate neighbor comfort threshold $T=3$ for the $8 \times 8$ city is strictly a small city phenomenon, and higher values of $T$ are necessary for more pronounced aggregation in large cities. We also find that aggregation in a large city is highly sensitive to the 
combination of the disparate neighbor comfort threshold and the number of vacancies in a city, in particular that aggregation is an increasing function of vacancies when $T=3$ but is inversely correlated with vacancies when $T=4$. We also find a remarkable linear dependence of aggregation measures on the vacancy ratio in large cities.

This work was partially supported by NSF (grants DMS-0355180 and 0400370). 


\section{References}

Benenson, I., Or, E., Hatna, E., and Omer, I. (2006). Residential Distribution in the City Reexamined. 9th AGILE International Conference on Geographic Information Science (April 2006).

Bruch, E. and Mare, R. (2006). Neighborhood Choice and Neighborhood Change. American Journal of Sociology 112(3): 667-709. doi: 10.1086/507856.

BusinessLocate (2009). Occupancy ratio. Http://www.realestateagent.com/glossary/realestate-glossary-show-term-1699-occupancy-ratio.html.

Clark, W. (1986). Residential segregation in American cities: A review and interpretation. Population Research and Policy Review 5(2): 95-127. doi: 10.1007/BF00137176.

Clark, W. (1991). Residential Preferences and Neighborhood Racial Segregation: A Test of the Schelling Segregation Model. Demography 28(1): 1-19. doi: 10.2307/2061333.

Clark, W. (1992). Residential preferences and residential choices in a multiethnic context. Demography 29(3): 451-466. doi: 10.2307/2061828.

Clark, W. and Fossett, M. (2008). Understanding the social context of the Schelling segregation model. Proceedings of the National Academy of Sciences 105(11): 41094114. doi: 10.1073/pnas.0708155105.

Duncan, O. and Duncan, B. (1955). A Methodological Analysis of Segregation Indexes. American Sociological Review 20(2): 210-217. doi: 10.2307/2088328.

Fossett, M. (2006). Ethnic Preferences, Social Distance Dynamics, and Residential Segregation: Theoretical Explorations Using Simulation Analysis. The Journal of Mathematical Sociology 30(3): 185-273. doi: 10.1080/00222500500544052.

Gerhold, G., Glebsky, G., Schneider, C., Weiss, H., and Zimmermann, B. (2008). Computing the complexity for Schelling segregation models. Communications in Nonlinear Science and Numerical Simulation 13(10): 2236-2245. doi: 10.1016/j.cnsns.2007.04.023.

Laurie, A. and Jaggi, N. (2003). Role of 'Vision' in Neighbourhood Racial Segregation: A Variant of the Schelling Segregation Model. Urban Studies 40(13): 2687-2704. doi: $10.1080 / 0042098032000146849$.

Massey, D. (1990). American Apartheid: Segregation and the Making of the Underclass. American Journal of Sociology 96(2): 329-357. doi: 10.1086/229532.

Massey, D. and Denton, N. (1988). The Dimensions of Residential Segregation. Social Forces 67(2): 281-315. doi: 10.2307/2579183. 
Massey, D., White, M., and Phua, V. (1996). The Dimensions of Segregation Revisited. Sociological Methods \& Research 25(2): 172-206. doi: 10.1177/0049124196025002002.

Oliphant, T. (2006). A Guide to NumPy. Trelgol Publishing.

Pollicott, M. and Weiss, H. (2001). The Dynamics of Schelling-Type Segregation Models and a Nonlinear Graph Laplacian Variational Problem. Advances in Applied Mathematics 27(1): 17-40. doi: 10.1006/aama.2001.0722.

Portugali, J., Benenson, I., and Omer, I. (1994). Sociospatial residential dynamics: stability and instability within a self-organizing city. Geographical Analysis 26(4): 321-340.

Sander, R., Schreiber, D., and Doherty, J. (2000). Empirically Testing a Computational Model: The Example of Housing Segregation. Proceedings of the Workshop on Simulation of Social Agents: Architectures and Institutions pp. 108-115.

Schelling, T. (1969). Models of segregation. American Economic Review 59(2): 488-493. Papers and Proceedings of the Eighty-first Annual Meeting of the American Economic Association (May, 1969).

Schelling, T. (1971a). Dynamic models of segregation. Journal of Mathematical Sociology 1(1): 143-186.

Schelling, T. (1971b). On the ecology of micromotives. The Public Interest 25: 61-98.

Schelling, T. (2006). Micromotives and macrobehavior. New York: W.W. Norton.

Simon, B. (1993). The statistical mechanics of lattice gases. Princeton University Press.

Singh, A., Vainchtein, D., and Weiss, H. (2009). Schelling's Segregation Model with Minorities .

Vinkovic, D. and Kirman, A. (2006). A physical analogue of the Schelling model. Proceedings of the National Academy of Sciences 103(51): 19261-19265. doi: 10.1073/pnas.0609371103.

Weisbuch, G., Deffuant, G., Amblard, F., and Nadal, J. (2002). Meet, discuss, and segregate! Complexity 7(3): 55-63. doi: 10.1002/cplx.10031.

Young, H. (1998). Individual Strategy and Social Structure: An Evolutionary Theory of Institutions. Princeton University Press.

Zhang, J. (2004). Residential segregation in an all-integrationist world. Journal of Economic Behavior and Organization 54(4): 533-550. doi: 10.1016/j.jebo.2003.03.005. 
Singh et al:: Schelling's Segregation Model: Parameters, scaling, and aggregation 